\title{
$\mathbf{X}$.
}

\section{Zur Kenntniss der Osteopsathyrosis.}

\author{
(Aus dem Pathologischen Institut zu München.) \\ Von Dr. Eugen Enderlen, \\ ebem. Assistenten am Pathologischen Institut, jetzt Assistenzarzt der chir. Abth \\ des städtisschen Krankenhauses 1 . I. zu München.
}

(Hierzu Taf. VI. Fig. 1-2.)

Bei dem relativ seltenen Vorkommen der sogenannten Osteopsathyrosis dürfte es von einigem Interesse sein, einen hiezu gebörigen Fall mitzutheilen. Bevor ich denselben bespreche, möchte ich die in der Literatur vorliegenden Angaben, soweit sie zugänglich waren, anführen.

J. T. Lobstein, (Lebrbuch der pathologischen Anatomie, deutsch von Neurohr 1835) von welchem der Name Osteopsathyrosis ( $\psi \alpha \vartheta v \varrho o ́ s, z e r b r e c h l i c h$, mürbe) stammt, führt als Grund die von Curling so benannte excentrische Knochenatrophie an. „Die Markhöhle hat sich auf Kosten der compacten Substanz ausserordentlich erweitert, die Wände dieser Höhle sind äusserst dünn und mürbe geworden, was denn auch ihre Leichtigkeit und Brüchigkeit erklärt." Fragilitas ossium wird nach ihm hauptsächlich bei Kindern und Greisen beobachtet; die Affection kann so stark sein, dass unbedeutende Ursachen hinreichen um Knochenbrüche zu veranlassen. Man sah 3 Kinder ans einer Familie, die sich binnen 5 Jahren bei unbedeutenden Fällen acht mal Arm und Bein brachen. Lobstein erwähnt Goodwin (Hufeland's Archiv der prakt. Heilkunde Bd. 25), der die Geschichte einer Frau anführt, die innerhalb $2 \frac{1}{2}$ Jahren 23 Knochenbrüche erlitt. Als Ursache wurde nur das vorgerückte Alter gefunden. - An den Knochen ist nach Lobstein keine Anschwellung, ausser nahe am Gelenk, es ist kein Anzeichen von erhöhter Thätigkeit, kein Uebermaass der Ernährung, sondern eher eine Verkümmerung, ein Trägheit des Ernährungsprozesses vorhanden. Nach Troja's Untersuchungen sei 
है

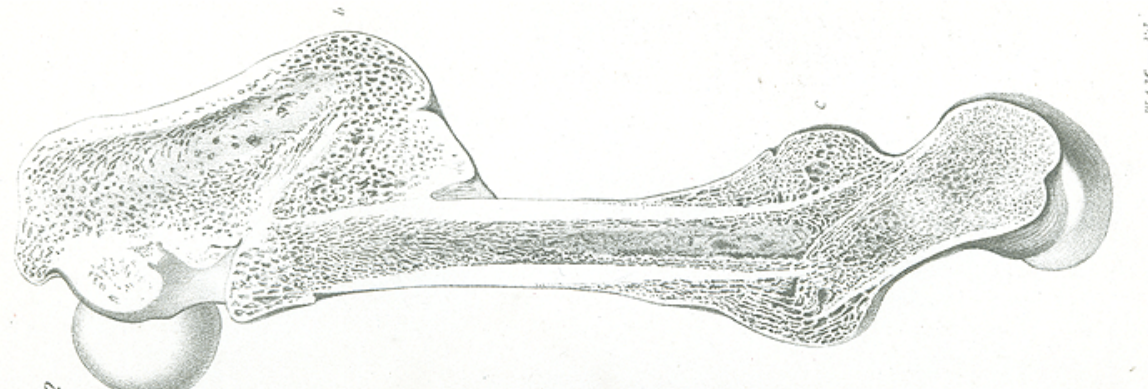

ה
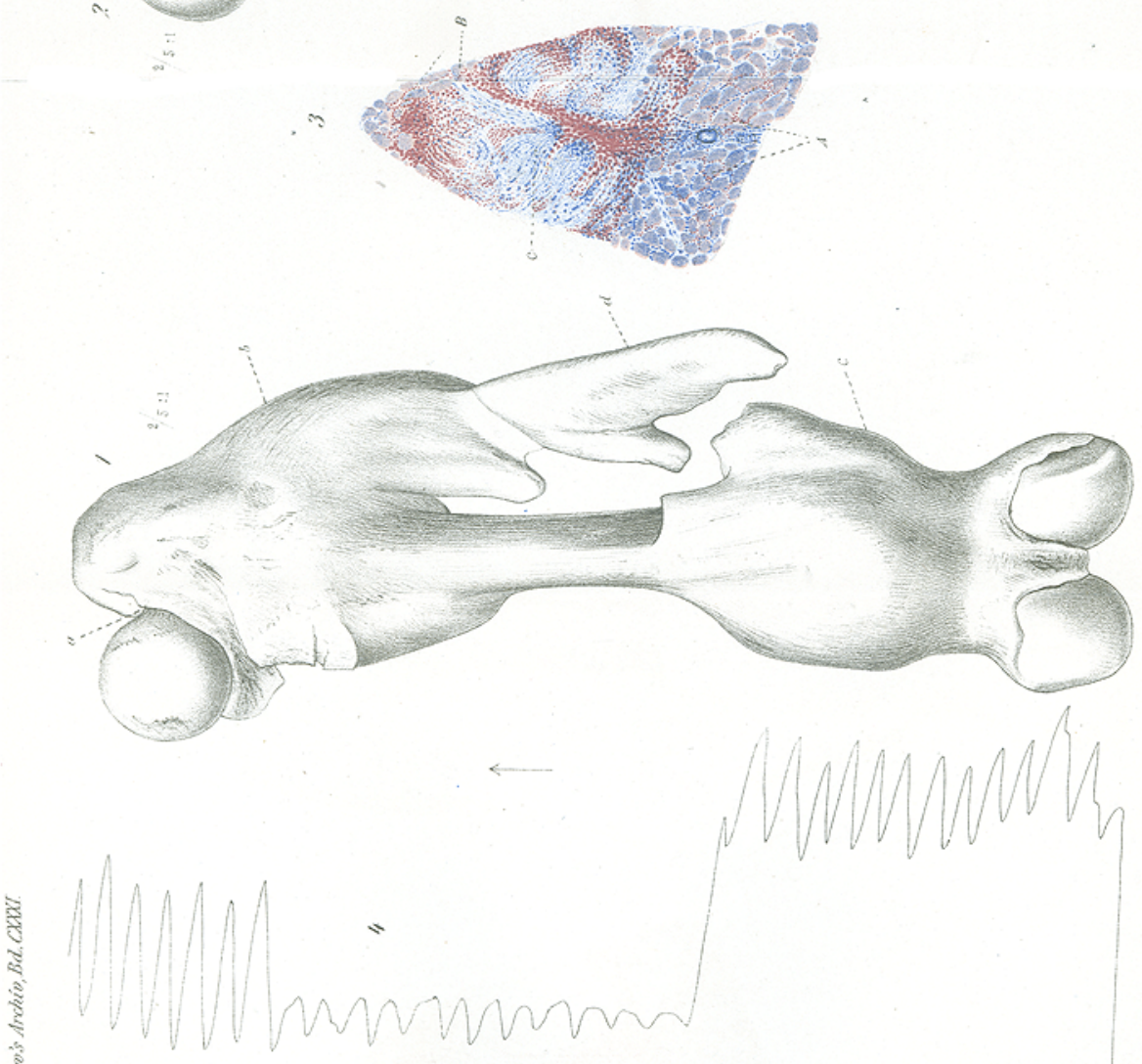
aber wieder die Knochenmürbigkeit weder vom Ueberfluss der phosphorsauren Kalkerde, noch von der Verminderung der Gallerte abhängig. - Die excentrische Knochenatrophie können wir nach dem Sectionsbefunde im vorliegenden Falle mit Sicherheit ausschliessen; die Markhöhle des erhaltenen Femurstückes war nicht erweitert, die Corticalis weder dünn, noch mürbe.

Im Lehrbuch von Pitha und Billroth wird Cap. XXXVIII von Volkmann angeführt, dass die Osteopsathyrosis nur ein sehr frappantes Symptom der an und für sich verschiedensten, die natürliche Fertigkeit und Widerstandsfähigkeit des Knochengewebes herabsetzenden Krankheiten sei. So können Rachitis, Osteomalacie und die Knochenatrophie eine mehr oder weniger grosse Disposition zu Fracturen unterhalten (nach Bruns käme hierzu noch die Tabes). Ausser diesen giebt es noch eine Osteopsathyrosis, deren Aetiologie bis jetzt vollkommen dunkel ist, bei der die Knochenbrüchigkeit das einzige Symptom bildet. Die Patienten sind sonst vollkommen wohl, ein leichter Stoss, eine rasche Bewegung genügt, um die Fractur herbeizuführen. Diese, die idiopathische Osteopsathyrosis, ist manchmal angeboren, dann bei einer Anzahl von Mitgliedern der betreffenden Familie; sie schleppt sich auch wohl an Intensität zunehmend, durch mehrere Generationen hin fort. Andere Male bildete sich die Krankheit ohne nachweishare Ursache erst später aus und bestand dann durchs ganze Leben, so dass die betreffenden $\mathrm{Pa}$ tienten sich eine Unmenge von Fracturen zuzogen, ja in Folge der dabei stattfindenden Uebereinanderschiebung der Fragmente ganz klein blieben, oder immer kleiner wurden. So wurde im Middlesex Hospital (London med. Gaz. Vol. XII. 1853. p. 366) ein 14 jähriges Mädchen behandelt, welches, obwohl sonst ganz gesund, seit seinem 3. Jahre bereits 31 Fracturen erlitten hatte.

Volkmann erwähnt ebenfalls dio rasche Heilung der Fracturen. Veranlassung zur Knochenbrüchigkeit ist nach Volkmann am wahrscheinlichsten eine Veränderung der knorpeligen Grundsubstanz der Knochen. In früherer Zeit wurde Osteopsathyrosis nicht selten in Folge von Scorbut beobachtet und man beobachtete zaweilen, dass der Callus bereits consolidirter Fracturen erweicht und resorbirt wurde.

Gurlt giebt eine Reihe von Beobachtungen an, bei welchen 
nie durch die Section ein anatomischer Beweis geführt werden kounte, $o b$ und welche Veränderungen die Knochensubstanz erlitten hatte.

Die Erblichkeit anlangend bringt er die Fälle von

O. J. Ekmann, (praes. J. G. and Diss. med. descriptionem et casus aliquot osteomalaciae sistens. Upsaliae 1788) der von einer schwedischen Familie mittheilt, dass bei ihr durch 3 Generationen Zwergwuchs herrschte, gepart mit einer eigenthümlichen Weichheit und Brüchigkeit der Knochen, die starke Verunstaltung bewirkte. Hiermit hat unser Fall keine Aehnlichkeit; erstens fehlt die Erblichkeit, zweitens die abnorme Weichheit und Biegsamkeit der Knochen. Aus ersterem Grunde sind auch die Beobachtungen von

Goddow (W. Gibson, Institutes and Practice of Surgery. 7. edit. Philadelphia 1845. Vol. I. p. 237) und

F. Pauli (zu Landau), Untersuchungen und Erfahrungen im Gebiete der Chirurgie, Leipzig 1844, $8 \mathrm{~m}, 4$ Tafeln S. 35) auszuschliessen.

Ebenso weisen die Mittheilungen von

Axmann (zu Wertheim), Annalen f. d. ges. Heilk., red. von d. grossherzoglich Badischen Sanitäts-Commission, Jahrg. 4. H. 1. 1831. S. 58. Lond. med. Gazette (Vol. XII. 1833. p. 366) Differenzen auf, indem hier mehrere Kinder einer Familie an Osteopsathyrosis litten ${ }^{1}$ ). Gurlt glaubt hier ein Analogon zu der noch viel des Räthselhaften bietenden erblichen Neigung zu Blutungen zu haben.

Bei Jacquinelle (Journ. de Medec. chir. Pharm. T. 77. 1788), traten die Fracturen schon im 12. Jahre auf und erstreckten sich auf die Arme und die. Oberschenkel.

Der Patient von W. Gibson (Institutes and Practice of Surgery 7. edit. Vol. I. p. 234) hatte im Alter von 23 Jahren bereits 24 Fracturen erlitten (Oberarm, Vorderarm, Ober- und Unterschenkel und beide Schlüsselbeine).

F. Mettauer's 70 jähriger Mann war von Jugend auf zu Knochenbrüchen geneigt; Ulna, Radius, Humerus, Claviculae, Tibia, Fibula, Femur, Costae. (Immer erfolgte sehr rasche Heilung der Fracturen.)

1) Die Geschwister unseres Patienten waren davon verschont. 
Bei einem 10 jährigen Knaben sah Earle (Ed. Stanley, A Treatise on Diseases of the Bones p. 240) 8 Fracturen, 6 an der einen Tibia, 2 am Femur.

Tyrell († St. Thomas' Hosp. Reports No. 1. Nov. 1835) sah bei seinem Falle 22 Fracturen, die alle sehr rasch heilten.

Mayo führt (Lond. Med: and Surg. Journ. Vol. V: 1834) Fracturen bei einem 41 jährigen Manne an; zwei davon hatte sich letzterer beim Anstossen der Zehe an eine Hervorragung des Strassenpflasters zugezogen.

Houston (Dublin Journ. of med. and chem. sc. Vol. VIII. 1836. p. 474) beobachtete bei einem gesunden 5 jährigen Knaben 4 Fracturen innerhalb zweier Jahre, jedesmal nach einem unbedentenden Falle; ferner citirt er eine Mittheilung von Kirby, der bei einem Manne "Fracturen fast aller langen Knochen“ nach geringgradigen Veranlassungen sah.

Mebes (v. Mebes, Die Knochenbrüche. Leipzig 1845) beschreibt einen Mann (Potator), der sich ausser Hand- und Fusswurzelknochen nur noch den 1 . Oberschenkel und r. Oberarm noch nie fracturirt hatte. Die Heilung beanspruchte hier längere Zeit als sonst.

Velpeau erwähnt (Gaz. des Hôp. 1847. p. 265) ein 15 jähriges Mädchen das 6 Fracturen sich zugezogen hatte, 2 davon beim Gehen!

Lange (Günzburg's Zeitschrift für klin. Medicin, Jahrg. III. 1852. p. 263): 11jähriges Mädchen mit scrofulösem Habitus, das nach und nach 7 Knochenbrüche erlitt.

Willaw Parker (New-York Journal of Medic. 1852): kleines schwächliches Mädchen, das nach geringen Traumen schon Eracturen erlitt. (Rechte Tibia, rechten Femur, Clavicula.)

F. Wakeley (Lancet 1856. Vol. I. p. 43) : kräft. Mann. Ruptur des Lig. patellae (Muskelaction). Fractur des r. Femur (beim Gehen, ]. Fibula beim Gehen).

Chadwich (Association Journ. 1854. Jan. Febr. March und Gaz. méd. de Paris 1854. p. 539). Junger schwächlicher Mann, Syphilis, r. Humerus, 1. Humerus, r. Femur bei kleinen Muskelanstrengungen. Innerhalb $7-8$ Jahren regelmässig ein Glied gebrochen. Schlechte Heilung.

Lancet 1827 (Beylard): 32 jähr. Mann, r. Unterschenkel, 
r. Oberschenkel, $5 \mathrm{mal} \mathrm{l}$. Unterschenkel $3 \mathrm{mal}$, r. Humerus $3 \mathrm{mal}$, I. Humerus 1 mal. Alle Brüche nach leichter Gewalteinwirkung. 50 jähr. Mann erlitt nach einer Fractur des l. Oberschenkels mit 21 Jahren später bei den geringsten Veranlassungen Brüche. l. Oberschenkel 6 mal, 3 mal r. Femur, 2 r., 1 l. Tibia.

Fock (Deutsche Klinik 1855. S. 314), 28 jähr. Mann bei Fall Coll. hum. dextr., bei ähnlicher Gelegenheit Fr. hum. sinistr.; bei rascher Armbewegung Fr. colli hum. anal. dextr., Fract. hum. dextr. ohne Ursache, Fract. fem. geringe Veranlassung, langsame Consolidation. 1 mal Pseudarthrosenbildung (hum. sin.).

Auffallend ist nach Gurlt, wie schon früher erwähnt, der rasche Reparationsprozess. Eine ausgedehnte Knochenatrophie glaubt Gurlt nicht annehmen zu dürfen, da es bei den meisten Individuen hiess, sie hätten zwar einen schwachen Knochenbau gezeigt, seien aber sonst anscheinend ganz gesund gewesen; eine aus irgend welehen Ursachen erworbene Atrophie oder Rarefication der Knochen müsste nach Gurlt mit einer beträchtlichen Atrophie der Weichtheile verbunden sein. Die Annahme einer relativ zu grossen Ablagerung von Kalksalzen, welche die Sprödigkeit der Knochen bedingt, hält Gurlt für nicht bewiesen; er glaubt vielmehr, dass die grössere oder geringere Widerstandsfähigkeit der Knochen von der Compactheit ihrer Substanz abhänge.

Bruns (Lehre von den Knochenbrüchen, 1886) führt an, dass die Aetiologie der Osteopsathyrosis noch dunkel sei; in einzelnen Fällęn ist die abnorme idiopathische Knochenbrüchigkeit angeboren, zuweilen erstreckt sich dieser Einfluss der Heredität sogar durch mehrere Generationen hindurch (Ekmann!). In anderen Fällen trat sie in früher Jugend auf ohne hereditäre Disposition, zuweilen später und bestand das ganze Leben hindurch. Die Fracturen heilten nach Bruns in der gewöhnlichen Zeit, nur hie und da abnorm langsam.

Hamilton (Knochenbrüche und Verrenkungen) behandelte einen 53 jähr. Mann mit 11 Fracturen und 2 Luxationen; die Disposition zu beiden war in der Familie erblich.

Greenisch A. W. (A case of hereditary tendency to fragilitas ossium) schildert 2 Familien, in denen sich die abnorme Knochenbrüchigkeit durch 3 Generationen vererbte. 
Blanchard: $12 \frac{1}{2}$ jähr. Mädchen, seit 2 Lebensmonaten im Ganzen 41 Fracturen bei den leichtesten Gewalten; 14 Fracturen am rechten, 11 am 1 . Unterschenkel.

Arnott: 14 jähr. Mädchen, seit 3. Lebensjahr 31 Knochenbrüche, 9 am r. Unterschenkel, 7 am r. Oberschenkel.

Jones: Mulatte, 50 Knochenbrüche, ein Vetter desselben litt an derselben Krankheit.

Bruns, Spont. Fractur bei Tabes (Berliner klin. Wochenschrift No. 11. 1882), der 30 Fälle von Spontanfracturen bei Tabes zusammenstellte, theilt in einer eigenen Beobachtung mit, dass bei einer Frau von 57 Jahren vor 20 Jahren Tabes zu entwickeln sich begann; vor 3 Monaten Fractur des r. Vorderarmes, vor 6 Wochen beim Heben einer Schüssel l. Vorderarm; beide Fracturen heilten mit enormer Callusbildung; die rechts in 10 , die links in 6 Wochen.

Charcot fasst die Knochenbrüchigkeit als Folge einer durch die Spinalerkrankung bedingten trophischen Störung des Knochengewebes auf.

Aus den Sectionsberichten ist nach Bruns zu ersehen, dass die Veränderungen der Knochen denen der excentrischen Atrophie entsprechen.

Regnard (bei Bruns) fand anorganische Substanz 24 (66) pCt., organische 76 (33) pCt.

Die Abnahme der Knochenerde beruhte auf Verminderung des Gebaltes an Phosphaten, die Zunahme der organischen Bestandtheile auf einer enormen Vermehrung des Fettgehaltes, der 37 pCt. des Gewichtes betrug. Es handelt sich also um einen beinahe vollständigen Schwund der Kalksalze und Ersatz durch Fett, d. h. eine Rarefaction der compacten Substanz und Anfüllung der erweiterten Räume mit Fett. Man könnte demnach diese Form als eine neurotische Art der Osteopsathyrosis auffassen.

Maydl (Allgemeine Wiener Med. Zeitung. 1882. No. 31. 32) berichtet über einige Fälle von Spontanfracturen, von welchen aber nur einer hier interessirt. Mann, $27 \mathrm{~J}$, von schlechtem Ernährungszustand, hatte sich beim Bügeln eine Fractur des 1. Radius zugezogen, langsame Heilung. 3 Monate nach der ersten Fractur Bruch beider Knochen beider Vorderarme, langsame Consolidation. 
In unserem Falle, welcher mir von Prof. Bollinger zur Veröffentlichung überlassen wurde, handelt es sich um einen Mann von $61 \mathrm{Jahren}^{1}$ ). In der Familie desselben hatte niemand an abnormer Knochenbrüchigkeit gelitten. Patient selbst war in der Jugend gracil gebaut, wurde für einen Phthisiker gehalten. 1862 machte er eine Meningitis durch; seit dieser Zeit werden grosse Dosen Morphium subcutan aufgenommen. Winter 1890 litt er schwer an Influenza und konnte sich ron ihr nicht mebr erholen. Der Tod erfolgte October 1890. Die anatomische Diagnose ${ }^{2}$ ) lantete: Adipositas cordis, bepatis, allgemeine Fettsucht, Pachymeningitis fibrosa chronica, bedeutende Hyperostose des Schädeldaches, Fragilitas ossium, Hyperostose geheilter Fracturen der Schenkelknochen, chronischer Morphinismus.

Im Ganzen erlitt Patient, welcher zuletzt in Behandlung des Herrn Geheimrath Dr. von Kerschensteiner stand, 6 Fracturen, 3 am rechten Femur, 3 am linken. Der Zeit nach vertheilen sie sich auf Sommer 1873 (hier soll ein leichter Stoss die Ursache gewesen sein), Sommer 1879, Sommer 1880, Sommer 1882, Sommer 1883, Winter 1887. Mit Ausnahme des ersten Knochenbruches war nie ein Trauma die Ursache, es genügte eine -geringe Bewegung die Fractur hervorzurufen. Auffallend war der ausserordentlich schnell und leicht vor sich gehende Reparationsprozess der Fracturen; dieselben heilten vor der für Brüche an gesunden Knochen als ungefähre Norm angenommenen Zeit. Weiters bemerkenswerth ist, dass Patient immer einige Zeit vor dem Knochenbruche angab, er werde sich in Kurzem eine Fractur zuziehen. Bestimmend waren hiebei für ihn sehr starke Schmerzen im Stirnbein, entsprechend der grossen Fontanelle.

Der bei der Obduction herausgenommene rechte Oberschenkel bot folgenden Befund:

Gewicht $1730 \mathrm{~g}^{3}$ ). Länge von der Spitze des grossen Trochanter bis zur Gelenkfläche des Condyl. int. beträgt $39,7 \mathrm{~cm}$. Die Richtung des oberen Drittels geht von der Trochanterspitze in einer Länge von $15 \mathrm{~cm}$ schief nach vorn und unten und bildet mit der folgenden mittieren Partie einen nach unten und binten offenen stumpfen Wiakel. Das mittlere Stück, $4,5 \mathrm{~cm}$ lang, läuft ziemlich gerade nach abwärts, das unterste Drittel ist etwas nach binten geneigt. Der Trochanter im Ganzen verdickt, unterbalb desselben findet man eine bedeutende Auftreibung des Knochens in Birnform. Die

1) Der in Rede stehende Fall zeigte entsprechend einer hochgradigen Taubheit im Bereiche des Gehörorganes hocbgradigen Nervenschwund in sämmtlichen Windungen der Sebnecke (vergl. Bezold und Scbeibe, Ein Fall von hochgradigem Nervensebwund in sämmtlichen Windungen der Schnecke. Zeitschrift für Ohrenbeilkunde. Bd. XXII). Mit Notizen aus der Krankengeschichte und der Angabe, dass in den oberen Theilen der Schnecke auch Knochendefecte sich vorfanden, die als Theilerscheinung der allgemeinen Skeleterkrankung aufgefasst werden.

2) Sections-Journal des Patholog. Instituts. No. 648. 1890.

3) Femar eines ebenso alten und gleichgrossen Mannes wog $940 \mathrm{~g}$. 
stärkste Hervorwölbung ist $12 \mathrm{~cm}$ unterbalb der Trocbanterspitze. Hier theilt sie sich in zwei gabelförmig nach unten und innen weitergehende Aeste; der mediale Ast $3,6 \mathrm{~cm}$ lang, der laterale $2,8 \mathrm{~cm}$ lang. Der Winkel, welchen beide zusammen bilden, beträgt $40-45^{\circ}$. Am distalen Ende des lateralen Astes findet sich eine $3,5 \mathrm{~cm}$ breite, $2 \mathrm{~cm}$ lange, straffe Bindegewebsspange, an welche sich ein freies Knoehenstück anschliesst. Die grösste Länge beträgt $12,5 \mathrm{~cm}$, die grösste Breite $7 \mathrm{~cm}$, die Dicke $2 \mathrm{~cm}$. Der äussere Rand bildet einen seichten Bogen, mit der Concavität nach innen; der hintere Rand weist einen wenig gekrümmten Bogen auf, mit der Concavität nach rorn. Der obere Rand verlänft parallel dem lateralen Zacken; der innere Rand sieht leicht gekrämmt nach unten und aussen. Von der Vereinigungsstelle des oberen und medialen Randes läuft ein $5,5 \mathrm{~cm}$ langer, $6,3 \mathrm{~cm}$ dicker Knochenzacken schief nach innen und unten, am distalen Ende sich etwas verjüngend. Der Winkel, den beide Knochenstücke mit einander bilden, hat etwa $30^{\circ}$. An der hinteren Partie ist die Auftreibung bedeutend geringer, die Oberfiäche derselben ziemlich glatt. An der medialen Seite ist die Auftreibung wieder stärker, ebenfalls ziemlich glatt, und weniger steil, mehr langgezogen auf das gerade Stück übergebend Der Trochanter minor ist etwas vergrössert, die Linea intertrochanterica wird durch eine im rechten Winkel schneidende, leicbt gekrümmte, schnabelförmig gebildete, mit der Spitze nach oben stebende Knochenplatte unterbrochen; die Concavität derselben nach hinten und etwas nach oben; die Höhe von der Basis zur Spitze (das ganze als Dreieck genommen) $4 \mathrm{~cm}$, die Basis $5 \mathrm{~cm}$. Das ganze Stück in geringem Grade beweglich; die Kapselwandung mit der binteren Fläche und dem oberen Rande zum Theil fest durch bindegewebige Spangen verbunden.

Auf das gerade Stück folgt eine zweite, ebenfalls birnförmige Auftreibung, die jedoch nicht so hochgradig wie die erste ist. Der grösste Umfang derselben beträgt $26 \mathrm{~cm}$; die äussere Partie vollkommen glatt; die Fortsetzung der Linea aspera femoris ist nach innen und hinten durch 2 ziemlich stark rorspringende Knochenzacken gebildet.

Der erste Zacken beginnt gleich am Ansatze an das normale Stück des Femur, überragt das Niveau desselben um $1 \mathrm{~cm}$. An seinem unteren Ende setzt sich staffelförmig der zweite etwas grössere Zacken an, welcher der Knochenauftreibung direct aufsitzt, etwa in Kleinwallnussgrösse.

Nach unten fällt die Verdickung ziemlich steil ab. Vorn ist der Vebergang von dem oberen Ende der Gelenkfläche $2 \mathrm{~cm}$ entfernt; die Distanz von der Tuberositas condyl. ext. beträgt $2,2 \mathrm{~cm}$, von der des Condyl. int. $2,3 \mathrm{~cm}$. Die beiden Condylen und deren Gelenkfläche sind ohne Befund.

Der Gelenkkopf zeigt an seiner vorderen Partie nabe dem Uebergang auf den Hals eine $3,5 \mathrm{~cm}$ lange, $0,5 \mathrm{~cm}$ breite, flache Usur, die freigelegten Partien fühlen sich rauh an; der Hals ziemlich kurz, misst $2 \mathrm{~cm}$. Die Verbindung mit dem Oberschenkelknochen ist nicht fest, sondern gelenkig. Von dem lateralen Ende des Halses zieht ein derbes, durch aufgelagerte, sehnig glänzende Streifen verstärktes Band zur Kapsel. Unterbrochen wird dasselbe durch einen an der Vorderseite verlaufenden sagittalen Schlitz; die Länge 
desselben 3,0 cm, grösste Breite $0,8-0,9 \mathrm{~cm}$. Der Rand der Oeffnung wellenförmig; durch denselben sieht man ein nengebildetes Gelenk, dessen eine Bälfte von der Fracturstelle des Schenkelbalses, die andere Hälfte von der Fossa trochanterica gebildet wird. Die Gelenkflächen sind von einer dünnen Knorpelschicht überzogen, bieten ein welliges Aussehen. In der Tiefe sieht man ein etwa bohnengrosses, in geringem Grade bewegliches, ebenfalls überknorpeltes Knochenstück.

Am freien Rande der Fossa trochanterica, ebenso am freien Rande des Halses finden sich stecknadelkopfgrosse, bis fast linsengrosse warzenförmige Anflagerungen.

Der Knochen schneidet sich mit Ausnahme der mittleren normalen Partie äusserst weich. Auf dem Durchschnitte (Trochanterspitze und Mitte der Fossa poplitea) sieht man von der Vorderfläche des Trochanter nach abwärts zu eine zuerst $0,6 \mathrm{~cm}$ breite Lamelle compacter Knochensubstanz, nach abwärts ziehen. Sie beginnt $3,3 \mathrm{~cm}$ unterhalb der Trochanterspitze; $7,5 \mathrm{~cm}$ unterbalb der letzteren finden sich kleine, allmählich an Zahl zunehmende, unter einander communicirende Hohlräume, welcbe die Lamelle verdünnen. Ueber der grössten Ausdehnung des Callus ist nur eine papierdïnne Knochenlamelle. Vom Trochanter minor zieht in einer Länge von $3,0 \mathrm{~cm}$ schief nach vorn und unten eine zweite compacte Knochenleiste, an der breitesten Stelle $0,4 \mathrm{~cm}$ messend; sie bildet mit der Verlängerung des normalen Stückes einen Winkel von ungefähr $60^{\circ}$. Die beiden Leisten entsprechen offenbar der Corticalis des nach vorn und unten dislocirten proximalen Fracturendes. Von der hinteren Knochenlejste erstreckt sich zur vorderen Fläche des unteren Brucbstückes ein nur aus Spongiosa bestehender Callus. In denselben hinein lässt sich genau die compacte Knochensubstanz des unteren Brucbstückes verfolgen, jedoch verschmälert sich dieselbe zusehends nach oben, um kurz vor Erreichung des oberen Endes vollständig in Spongiosa sich aufzulösen. An der normaIen Stelle ist die Corticalis $0,5 \mathrm{~cm}$ dick; dann spaltet sich die vordere Lamelle in zwei Leisten. Die vordere, $2,5 \mathrm{~cm}$ lange, $1 \mathrm{~mm}$ dicke Wandung geht auf den früher erwähnten, unteren Callus über; die hintere verläuft langsam sich verjüngend als Fortsetzung der erhaltenen Partie gerade nach abwärts, bis in die Höbe des grössten Umfangs des unteren Callus. Parallel mit ibr verläuft die hintere Röbrenwandung ebenfalls mebr und mehr sich verschmälernd; umscblossen ist sie von Callusmasse, die von oben nach unten rasch an Consistenz abnimmt. Beide Lamellen bilden mit den von unten. kommenden, äusserst schmalen Leisten des unteren Drittels einen nach vorn offenen stumpfen Winkel. Die vordere Partie des unteren Brucbstückes ist in das obere eingekeilt, die vordere Wandung ist nahezu bis in die Mitte der Markhöble zu verfolgen. Die Verkürzung des Femur dürfte $8-10 \mathrm{~cm}$ betragen.

Oberer, unterer Callus und das frei pendelnde Knochenstück schneiden sich verhältnissmässig weich, ungefähr wie trockenes Brot, die mittlere erhaltene Knochenpartie ist dagegen sehr hart.

Schnitte aus den äusseren Partien des Callus zeigen die zufällig mitgetroffene Musculatur gut erbalten; die äussere Periostschicht kern- und ge- 
fässarm, die innere zeigt reichliche Gefässe und Kerne. Die Gefässe sind ziemlich gut gefüllt. Die an das Periost sich anschliessende Knochenlamelle ist ziemlich schmal. Knochenbälkchen von $0,025-0,126 \mathrm{~mm}$ Breite sind nach innen zu ziemlich ungleichmässig angeordnet, in ibnen finden sich ziemlich spärlich Knochenkörperchen. Das Mark besteht vorwiegend ans Fettmark, unregelmässig eingestreut in kleineren und grösseren Haufen ist lymphoides Mark; in letzterem wenig Riesenzellen, kleinere und grössere rundliche Zellen mit hellem Kerne. Ausserdem Pigment von gelblicher Farbe, meist in kleinen Häufchen beisammenliegend:

Knocbenscbliffe mit Ammoniak-Carmin gefärbt, ergeben eine schmale, ziemlich intensiv gefärbte Zone osteoiden Gewebes. Den letzterwähnten Saum finden wir auch bei dem freien Knochenstücke, welches ziemlich schmale Knochenbälkchen und reichlich Fettmark aufweist. Ich glaube, dass dieses Stück von dem oberen Callus abgesprengt wurde; hiefür spricht die geringe Entfernung von demselben, die bindegewebige Verbindung und der parallele Verlauf des oberen Endes mit dem unteren Callusende.

Der starke Knochenschwund sowobl in dem Appendix als auch in den beiden Callusmassen ist wohl daraus zu erklären, dass diese Stücke zum grossen Theile für die stützende Function des Knochens bedeutungslos wurden. Diejenigen Partien, welche einern Drucke noch ausgesetzt waren, sehen wir (wie auch auf der Zeichnung angegeben) mit reichlicheren stärkeren Knochenmassen versehen. Dass anch bei letzteren doch eine ziemlich starke Resorption stattfand, wird begreiflich, wenn man bedenkt, dass Patient während der letzten Lebensjahre stets gefahren wurde.

Schnitte aus dem erhaltenen Knochenstücke ergeben keinen Befund.

Die Wirbelsäule erinnerte beim Einschneiden wegen ibrer Weichheit an die von Ribbert beschriebene senile Osteomalacie (dieses Archiv Bd. 80 S. 436 ff.). Bei Färbung mit Ammoniak-Carmin sieht man schon bei schwacher Vergrösserung einen roth gefärbten Saurn von verschiedener Breite, scharf gegen den Markraum abgesetzt, weniger deutlich gegen die kalkhaltigen Partien. Die Grenze gegen die letzteren ist ziemlich unregelnässig, Hervorwölbungen wechseln mit flacheren oder tieferen Buchten ab. Die osteoide Zone enthält nur sehr wenig Knochenlsörperchen, der Form nach ähnlich den Kernen glatter Muskelfasern; Ausläufer sind nur sehr schwer sichtbar, manchmal finden sich feine Linien, welche dem rothen Rande parallel verlaufen; letztere werden von Ribbert ebenfalls als Knochenkörperchen aufgefasst.

Das Mark bestebt aus reichlichen Fettzellen, oft dicht beisammenstebend, obne dazwischen liegende Markzellen, an anderen Stellen sind zwischen dem Fette unter einander zusammenhängende Markzellenzüge, bald breiter, bald schmäler. Die Markzellen ein - und mebrkernig; daneben spärliche Riesenzellen, rothe Blutkörperchen, einzelne kernhaltige, kleine gelbbraune Pigmentbäufchen; die Gefässe sind allentbalben gut gefüllt. In den letzteren Punkten differiren die Befunde von Ribbert, der bei seinem hochgradigen Falle bedeutende Vermehrung der Markzellen fand, die durch ihre Menge gegenüber den Fettzellen das ganze Gesichtsfeld beherrschten. 
Das schwindende Knochengewebe wurde in unserem Falle durch massenhaft auftretende Fettzellen ersetzt, das Markfett besass eine ziemlich flüssige ölige Beschaffenheit, beim Durchsägen des Knochens floss es in grossen Tropfen ab. Bei der allgemeinen hochgradigen Fettsucht konnte man nach Volkmann wohl auch an eine Lipomanie der Knochen denken und den Knochenschwund als das Secundäre annehmen, doch glaube ich, dass die erste Ansicht zutreffender ist.

Die Analyse der Knocben wurde im physiologischen Institut von Herm Professor Tsuboi ausgeführt. Sie ergab:

\begin{tabular}{|c|c|c|c|}
\hline Stirnbein & . & $\begin{array}{l}\text { organisch in pCt. } \\
\quad \cdot 36, \pm 2\end{array}$ & $\begin{array}{c}\text { Asche in pCt. } \\
63,58\end{array}$ \\
\hline Schläfenbein & . & . . 36,19 & 63,81 \\
\hline Wirbelsäule & - & . . 54,70 & 45,30 \\
\hline Oberschenkel & . & . $\quad 37,77$ & 62,23 \\
\hline (Oberschenkel & normal) & 1) $\cdot 31,5$ & 68,5 \\
\hline Callus Obersch & enkel. & - . 44,69 & $55,31$. \\
\hline
\end{tabular}

Zieht man die von verschiedenen Seiten für die abnorme Knochenbrüchigkeit angegebenen ätiologischen Momente in $\mathrm{Be}$ tracht, so fallen, wie wohl aus dem Vorhergehenden ersichtlich ist, Scorbut, Rachitis und Osteomalacie weg. Ebenso ist nach der klinischen Beobachtung Tabes oder irgend welche andere Spinalerkrankung auszuschliessen.

Auch die chemische Analyse ergab keinen Anhaltspunkt für die Brüchigkeit. Das erhaltene Femurstück (mit diesem müssen wir ja hier rechnen) ergab gegenüber einem normalen nur ein Plus von 6,27 pCt. organischer Substanz.

Es handelt sich demnach wohl um die schon von Lobstein erwähnte idiopathische Form der Osteopsathyrosis, bei deren Entstehung vielleicht der mehrere Jahrzehnte hindurch dauernde excessive Morphiumgebrauch eine Rolle gespielt haben dürfte.

Herrn Obermedicinalrath Prof. Bollinger für die gütige Ueberlassung des Falles und für die vielfache Unterstützung meinen wärmsten Dank.

\section{Erklärung der Abbildungen.}

Taf. VI. Fig. $1-2$.

Fig. 1. Rechter Oberschenkel. a Collumfractur mit Pseudoarthrose geheilt. b Fractur im oberen Drittel. c Fractur im unteren Drittel. d abgesprengtes Stück, durch eine bindegewebige Spange mit dem Femur noch im Zusammenhange.

Fig. 2. Durchschnitt des Femur. b und c die Fracturstellen. 